\title{
COMPUTATIONAL METHOD FOR THE ANALYSIS OF DISSEMINATION OF SURFACE POLLUTION IN HYDROTECHNICAL RAMPARTS
}

\author{
Iryna Vergunova \\ Department of mathematical informatics \\ Taras Shevchenko National University of Kyiv \\ 64/13 Volodymyrska str., Kyiv, Ukraine, 01601 \\ vergunova@hotmail.com
}

\begin{abstract}
The conducted researches served as the basis for obtaining difference scheme for numerical realization of the two-dimensional model of mass transfer of a pollutant in the aeration zone of the soil of the agrolandscape with a piecewise smooth surface under the condition of instantaneous deposition of the pollutant onto the surface (as an example, Cs137 was taken as a pollutant on the basis of its passive behavior in the ground and the availability in the considered ground areas of agricultural uses due to the Chernobyl accident).

The properties of differential operators of the model and their difference analogues were studied, which allowed to substantiate the cost-effective difference scheme for the numerical solution of the problem of pollutant migration for given agrolandscape. The correctness and efficiency of the constructed two-layer implicit difference scheme is shown. This allowed to switch to the use of a chain of one-dimensional implicit tasks, in which the transition from one layer to another occurs in two steps. Obtained general computing costs allowed to assert that the proposed schemes are cost-effective difference schemes.

In turn, the use of an economical difference scheme made it possible to construct a method for the practical determination of the presence of a process of water erosion in the system of hydraulic rampart-terrace.
\end{abstract}

Keywords: mass transfer, two-dimensional model, difference scheme, soil aeration zone.

\section{Introduction}

The study of dissemination of anthropogenic pollution in the environment is an important scientific and applied task. Different approaches and methods are used to solve the problems of mass transfer of pollutants in the soil. The most popular in practical research are those that use as mathematical models of the regression equation and differential equations based on the use of physical laws related to the movement of moisture and heat flows.

The first class of models determines the existence of a large number of experimental data and has the limitations inherent in all regression models. Such models provide solutions with some reliability only at a certain interval $[1,2]$. Moreover, the required amount of data can not always be obtained. But the simplicity of computations in comparison with the calculations of models in which the differential equations are numerically solved and some formulation of tasks in the research make possible the widespread use of such models.

Physically justified differential models are based on the use of the laws of continuity and transfer. The flow of substances associated with diffusion, convection and dispersion movements, sorption and written as evolutionary differential equations [1,3]. Such models can be used in wider ranges, they give short and medium-term forecasts for different boundary conditions but it is necessary to apply numerical methods for their realizations. To simplify the calculations are often limited to the consideration of the formulation of such statements of tasks that suggest the existence of an analytical solution. This is a rather narrow class of tasks. Moreover, with a view to simplifying numerical implementations in such models, it is quite often considered moving of substances with soil moisture in only one profile using general theoretical equations with an exact analytical solution.

Given the relevance of studying the movement of pollutants in the aquatic environment, soil or air and the complexity of numerical implementation of the corresponding models, which including the analysis of the correctness and stability of the received difference schemes, studies 
related to the construction of economic schemes for numerical analysis of surface pollution are very important.

\section{Literature review and problem statement}

Determining the spread of anthropogenic pollution in the environment is an actual problems, therefore various tasks of mass transfer processes describing in several scientific publications. Among all publications should highlight research in agriculture and ecology, such as in [3-5]. Processes considered in these publications mostly are one-dimensional for horizontal or vertical profile and researchers use different methods to solve the problems of mass transfer. In these papers, the authors mainly use models derived from regression dependencies and models described by exact solutions of differential equations. For example, in [3, 5] various regression models, based on experimental findings, are shown. Methods using precise analytical solutions are proposed in [4] for various applications.

In [6] authors discuss the masstransfer problem of landscape for understanding the evolution of pollution distribution and soil erosion. In this paper examines the problem of horizontal landscape mass transfer within the vegetation in inhomogenous boundary surface. The paper points out that usually in the tasks of pollution transfer across soil objects, most of the researchers are considering the influence of the environment on the transfer process. The authors of the work aren't taken into consideration mechanical influence of boundary surface on the transfer process.

Important to practice tasks are tasks that take into account the vegetation in natural landscapes, as the results may allow to investigate pollution distribution soil erosion. A similar problem was considered in [7], but simplified boundary conditions were used to solve it.

The solutions used here are analytic, as in [8]. Moreover, these applied studies did not consider the numerical solution of tasks in the absence of analytical solutions.

Of course, to date, many different models of mass transfer of pollutants have been developed and used. This allows researchers to obtain answers for many tasks. Empirical models, also called data-driven models, have as its basis the experimental measurements of the transport of matter in a particular situation, which limits the class of problems for solution, since it makes it possible to only predict the development of the situation (for example, it occurs in numerous publications that study the migration of radionuclides along the vertical soil profile). The application of theoretical models (sciencebased models) in agricultural research tends to use the analytical solution of the convective diffusion equation for the vertical profile. But with this approach the predictive possibilities of solutions are also limited [1].

Due to the complexity of the processes occurring in the soil system, there is no single model of mass transfer processes. When constructing models of physico-chemical processes in soils, an approach based on the use of various modifications of mathematical models of moisture transport in the soil and the theoretically derived equations expressing the fundamental properties of the soil was widely used. Based on this approach, a general conceptual scheme was developed that is widely used in modern works and underlies the computer implementations of these processes.

Models of mass transfer of matter and heat in the ground based on the equation of convective diffusion in conjunction with the equations of hydromechanics and kinetic equations are common in theoretical studies. Such models try to simplify by reducing their dimension, averaging the desired value by one or two spatial coordinates. Due to the fact that the redistribution of pollutants in the soil is associated with the redistribution of moisture in it, often the focus is on the forecast of the groundwater regime, and the evaporation and movement of moisture in the aeration zone are taken into account by introducing the respective members.

Researchers are also using equations for describing the movement of various forms of moisture in the zone of soil aeration to study the propagation of soluble substances at small time intervals. Partly avoids such problem of the equation of mass transfer in the soil, in which it is difficult to obtain only the values of an effective diffusion coefficient $[2,9,10]$.

The use of the theoretical approach to the construction of models allows to describe the dynamics of the process, and the application of numerical methods significantly expands the class of solved problems, but in practice the process of solving the problem is often complex. This approach is used, for ex- 
ample, in $[1,2,9,11]$. The models used in these studies are spatially distributed dynamic models, which allow one to investigate mass transfer processes. Such studies suggest the use of numerical methods: the finite element methods, as in [12], or finite-difference schemes, as in [13-15]. But the complexity of the numerical realization leads to the fact that in these papers the authors also confine themselves to the consideration of two-dimensional domains with a piecewise smooth boundary.

In [13], the Crank-Nicolson difference scheme method is proposed to obtain a numerical solution, but the question of the existence of a solution of the differential problem is not considered. That models and numerical methods authors used only for estimating of soil pollution.

Analysis of scientific publications has shown that in almost all similar studies numerical solutions of problems are constructed, but the question of the existence of a solution of the problem and the correctness of the numerical solution for applied research is often remains open.

\section{The purpose and objectives of the study}

In this paper, the ways to solve the problem of mass transfer of a pollutant for agrolandscapes with a piecewise smooth surface where surface contamination occurred at some point are developed. Solution of this problem makes it possible to estimate the propagation of pollutant in the soil agrolandscape aeration zone and to determine the presence and extent of erosion in this agrolandscape.

The objects of our research are:

- the mathematical model of mass transfer in a bounded area with a piecewise-continuous surface, taking into account the nature of contamination and the existence of a unique solution of the corresponding differential problem;

- the difference analogue of a differential operator and the construction of a cost-effective difference scheme for the numerical solution of the problem of pollutant migration in the aeration zone of a given agrolandscape.

\section{The two-dimensional problem of pollutant migration under the condition of instantaneous contamination of the agrolandscape surface}

The determination of changes in the distribution of the concentration of soil pollutants is important for many modern studies, which require an analysis of the state of contamination zones. The study of the redistribution of a long-lived, poorly soluble solid pollutant, the migration of which occurs exclusively with the flow of moisture, also makes it possible to estimate the degree of water erosion in contaminated area.

\section{1. The mathematical model of pollutant migration and the existence of a solution of}

\section{the differential problem}

The problem of mass transfer in the zone of soil aeration in the system of rampart-terraces is investigated, therefore the process was considered taking into account its independence from the water regime of the soil. It is accepted that the vertical migration of the substance occurs convective with the flow of moisture through the soil profile. The fall of the pollutant on the soil surface was considered instantaneous. It is assumed that the profile of the soil was homogeneous with respect to depth and time, the filtration flow was linear, and the water saturated soil was isotropic, porous and not compressed. It is also admitted that the transfer process in the soil solution obeys Fick's law, therefore the equation of mass transfer $[10,15]$ are considered, on the basis of which the two-dimensional model of mass transfer in a bounded domain with a complex piece-smooth surface was investigated. In it: the effective diffusion coefficient is described by the function $\mathrm{D}(\mathrm{x})$ dependent on the spatial argument; the velocity of the directed diffusion displacement is constant $\mathrm{V}$.

Our mathematical model has the following form:

$$
\begin{aligned}
& \mathrm{Zu} \equiv \frac{\partial \mathrm{u}(\mathrm{x}, \mathrm{t})}{\partial \mathrm{t}}+\mathrm{Lu} \equiv \\
& \equiv \frac{\partial \mathrm{u}(\mathrm{x}, \mathrm{t})}{\partial \mathrm{t}}-\sum_{\mathrm{i}=1}^{2} \frac{\partial}{\partial \mathrm{x}_{\mathrm{i}}}\left(\mathrm{D}(\mathrm{x}) \frac{\partial \mathrm{u}(\mathrm{x}, \mathrm{t})}{\partial \mathrm{x}_{\mathrm{i}}}\right)+\sum_{\mathrm{i}=1}^{2} \mathrm{~V} \frac{\partial \mathrm{u}(\mathrm{x}, \mathrm{t})}{\partial \mathrm{x}_{\mathrm{i}}}=-\lambda \mathrm{u}_{0} \phi(\mathrm{x}) \delta(\mathrm{t}-0)
\end{aligned}
$$




$$
\begin{gathered}
\mathrm{u}(\mathrm{x}, 0)=0, \mathrm{x} \in \Omega, \\
\left.\left(-\sum_{\mathrm{i}=1}^{2} \mathrm{D}(\mathrm{x}) \frac{\partial \mathrm{u}}{\partial \mathrm{x}_{\mathrm{i}}}+\mathrm{Vu}\right)\right|_{\mathrm{x} \in \Gamma}=\mathrm{k}(\Gamma) \mathrm{c}_{0} \mathrm{q}_{0}(\mathrm{x}), \mathrm{t} \in[0, \mathrm{~T}], \\
\left.\left(-\sum_{\mathrm{i}=1}^{2} \mathrm{D}(\mathrm{x}) \frac{\partial \mathrm{u}}{\partial \mathrm{x}_{\mathrm{i}}}+\mathrm{Vu}\right)\right|_{\mathrm{x} \in \partial \Omega \backslash \Gamma}=0, \quad \mathrm{t} \in[0, \mathrm{~T}]
\end{gathered}
$$

in a limited area $\mathrm{Q}=\Omega \times(0 \leq \mathrm{t} \leq \mathrm{T})$, where $\Omega \subset \mathrm{R}^{2}$ with a piecewise smooth border $\partial \Omega ; \mathrm{D}(\mathrm{x})$ the function that is continuously differentiated in $\bar{\Omega}$, it inalienable and limited in the relevant area; $\mathrm{V}$ - the constant, $\mathrm{V}>0 ; \lambda$ - half-life coefficient; $\phi(\mathrm{x})$ - the function that describes the surface, that is, the upper boundary $\mathrm{G}$ of $\mathrm{W}, \phi(\mathrm{x}) \in \mathrm{L}_{2}(\Omega) ; \mathrm{u}_{0}$ - contamination; $\mathrm{k}(\Gamma)=\mathrm{k} \cos (\alpha(\Gamma))$, $\mathrm{k}-$ conductivity coefficient for the surface, $\alpha(\Gamma)$-slope of the surface of the terrace; $\mathrm{q}_{0}$ - the function that describes the flow of water with atmospheric precipitation with concentration $\mathrm{c}_{0} ; \mathrm{u}(\mathrm{x}, \mathrm{t})-$ the concentration of substance at the point $\widetilde{o}=\left(\mathrm{x}_{1}, \mathrm{x}_{2}\right) \in \bar{\Omega}$ at the moment $\mathrm{t}$.

Let's denote $\mathrm{D}(\mathrm{Z})$ the set of functions $\mathrm{u}(\mathrm{x}, \mathrm{t})$ twice continuously differentiated in $x$ in $\bar{\Omega}$ and continuously differentiated in $t$ on $[0, T]$ which satisfy the conditions $(2),(3)$. The set $\mathrm{D}(\mathrm{Z})$ is dense in space $L_{2}(Q)$, which makes it possible to determine in space $L_{2}(Q)$ of a linear operator $\mathrm{Zu}$ with a domain of definition $\mathrm{D}(\mathrm{Z})$.

Let's consider the space $\mathrm{H}$ with the norm

$$
\|\mathrm{u}\|_{\mathrm{H}}=\int_{\mathrm{Q}}\left(\mathrm{u}_{\mathrm{t}}^{2}+\sum_{\mathrm{i}=1}^{2}\left(\frac{\partial \mathrm{u}}{\partial \mathrm{x}_{\mathrm{i}}}\right)^{2} \mathrm{dQ}, \mathrm{u} \in \mathrm{D}(\mathrm{Z}) .\right.
$$

Then for any $u \in D(Z)$

$$
\|\mathrm{u}\|_{\mathrm{H}} \geq \mathrm{c}\|\mathrm{u}\|_{\mathrm{L}_{2}(\mathrm{Q})}, \quad \mathrm{c}=\mathrm{const}>0 .
$$

The proof. Given the norms above

$$
\|\mathrm{u}\|_{\mathrm{H}}=\int_{\mathrm{Q}}\left(\mathrm{u}_{\mathrm{t}}^{2}+\sum_{\mathrm{i}=1}^{2}\left(\frac{\partial \mathrm{u}}{\partial \mathrm{x}_{\mathrm{i}}}\right)^{2} \mathrm{dQ} \geq\left\|\mathrm{u}_{\mathrm{t}}\right\|_{\mathrm{L}_{2}(\mathrm{Q})} .\right.
$$

Since $u \in D(Z)$, then

$$
|\mathrm{u}(\mathrm{t}, \mathrm{x})| \leq \sqrt{\mathrm{T}}\left(\int_{0}^{\mathrm{t}} \mathrm{u}_{\tau}^{2} \mathrm{~d} \tau\right)^{1 / 2}
$$

and

$$
\max _{t \in[0, T]}|u(t, x)| \leq \sqrt{T}\left(\int_{0}^{T} u_{t}^{2} d t\right)^{1 / 2} .
$$

Therefore

$$
\left|\max _{\mathrm{t} \in[0, \mathrm{~T}]} \mathrm{u}(\mathrm{t}, \mathrm{x})\right|_{\mathrm{L}_{2}(\Omega)}^{2} \leq \sqrt{\mathrm{T}} \iint_{\Omega}^{\mathrm{T}} \mathrm{u}_{\mathrm{t}}^{2} \mathrm{dtd} \Omega=\sqrt{\mathrm{T}}\left\|\mathrm{u}_{\mathrm{t}}\right\|_{\mathrm{L}_{2}(\mathrm{Q})}^{2}
$$

Since the space $C[0, T]$ is embedded in $\mathrm{L}_{2}[0, \mathrm{~T}]$, then

$$
\left\|\mathrm{u}_{\mathrm{t}}\right\|_{\mathrm{L}_{2}(\mathrm{Q})}^{2} \geq \tilde{\mathrm{n}}\|\| \mathrm{u}\left\|_{\mathrm{C}\{0, \mathrm{~T}]}\right\|_{\mathrm{L}_{2}(\Omega)} \geq \mathrm{c}\|\mathrm{u}\|_{\mathrm{L}_{2}(\mathrm{Q}} .
$$

From (5) and (6) let's have (4). 
Operator

$$
\mathrm{Au}=-\sum_{\mathrm{i}=1}^{2} \frac{\partial}{\partial \mathrm{x}_{\mathrm{i}}}\left(\mathrm{D}(\mathrm{x}) \frac{\partial \mathrm{u}}{\partial \mathrm{x}_{\mathrm{i}}}\right)
$$

is symmetric in space $L_{2}(Q)$ but operators $A$ and $Z$ are not positively defined in space $L_{2}(Q)$. The conjugate problem has the form

$$
Z^{*} \mathrm{v} \equiv-\mathrm{v}_{\mathrm{t}}+\mathrm{Av}-\sum_{\mathrm{i}=1}^{2} \mathrm{~V} \frac{\partial \mathrm{v}}{\partial \mathrm{x}_{\mathrm{i}}}=\mathrm{g}(\mathrm{x}, \mathrm{t})
$$

where

$$
\mathrm{v}(\mathrm{x}, \mathrm{T})=0, \mathrm{x} \in \Omega,\left.\mathrm{v}\right|_{\mathrm{x} \in \partial \Omega}=0, \mathrm{t} \in[0, \mathrm{~T}]
$$

Similarly (4) can get

$$
\|\mathrm{v}\|_{\mathrm{H}^{*}} \geq \mathrm{c}\|\mathrm{V}\|_{\mathrm{L}_{2}(\mathrm{Q})}, \quad \tilde{\mathrm{n}}=\text { const }>0
$$

for any $\mathrm{v} \in \mathrm{D}\left(\mathrm{Z}^{*}\right)$, where

$$
\|\mathrm{v}\|_{\mathrm{H}}=\int_{\mathrm{Q}}\left(\mathrm{v}_{\mathrm{t}}^{2}+\sum_{\mathrm{i}=1}^{2}\left(\frac{\partial \mathrm{v}}{\partial \mathrm{x}_{\mathrm{i}}}\right)^{2} \mathrm{dQ}\right) .
$$

Let's introduce the negative spaces: negative space $\mathrm{H}^{-}$with the norm

$$
\|g\|_{\mathrm{H}^{-}}=\sup _{\mathrm{u} \neq 0} \frac{\left|(\mathrm{u}, \mathrm{g})_{\mathrm{L}_{2}(\mathrm{Q})}\right|}{\|\mathrm{u}\|_{\mathrm{H}}}, \quad \mathrm{u} \in \mathrm{H}, \mathrm{g} \in \mathrm{L}_{2}(\mathrm{Q})
$$

and negative space $\mathrm{H}^{*-}$ with the norm

$$
\|f\|_{H^{*-}}=\sup _{v \neq 0} \frac{\left|(v, f)_{L_{2}(Q)}\right|}{\|v\|_{H}}, v \in H^{*}, f \in L_{2}(Q) .
$$

The right-hand side of equation (1) is a function of space $\mathrm{H}^{*-}, \mathrm{H}_{2}^{-}(0, \mathrm{~T}) \otimes \mathrm{L}_{2}(\Omega) \supseteq \mathrm{H}^{-}(\mathrm{Q})$. For any $\mathrm{u} \in \mathrm{H}$

$$
\|\mathrm{Zu}\|_{\mathrm{H}^{*-}} \leq \mathrm{c}_{1}\|\mathrm{u}\|_{\mathrm{H}}, \quad \mathrm{c}_{1}=\text { const }>0 .
$$

The proof. Let consider

$$
\left|(\mathrm{Zu}, \mathrm{v})_{\mathrm{L}_{2}(\mathrm{Q})}\right|=\left|\int_{\mathrm{Q}}\left(\mathrm{u}_{\mathrm{t}} \mathrm{v}+\mathrm{Auv}+\mathrm{Buv}\right) \mathrm{dQ}\right| \leq \mathrm{I}_{1}+\mathrm{I}_{2}+\mathrm{I}_{3},
$$

where

$$
\mathrm{Bu}=\sum_{\mathrm{i}=1}^{2} \mathrm{~V} \frac{\partial \mathrm{u}}{\partial \mathrm{x}_{\mathrm{i}}}
$$

Taking into account (4)

$$
I_{1}=\left|\int_{Q} u_{\mathrm{t}} \mathrm{vdQ}\right| \leq\left(\int_{\mathrm{Q}}\left|\mathrm{u}_{\mathrm{t}}\right|^{2} \mathrm{dQ}\right)^{1 / 2}\left(\int_{\mathrm{Q}}|\mathrm{v}|^{2} \mathrm{dQ}\right)^{1 / 2} \leq\|\mathrm{u}\|_{\mathrm{H}}\|\mathrm{v}\|_{\mathrm{L}_{2}(\mathrm{Q})} \leq \tilde{\mathrm{n}}\|\mathrm{u}\|_{\mathrm{H}}\|\mathrm{v}\|_{\mathrm{H}^{*}} .
$$


As

$$
\int_{Q} \mathrm{vAudQ}=\int_{\mathrm{Q}} \mathrm{v} \frac{\mathrm{d}}{\mathrm{dt}} \int_{0}^{\mathrm{t}} \operatorname{Aud} \tau \mathrm{d} \mathrm{Q}=-\int_{\mathrm{Q}} \mathrm{v}_{\mathrm{t}} \int_{0}^{\mathrm{t}} \operatorname{Aud} \tau \mathrm{d} \mathrm{Q},
$$

then

$$
\mathrm{I}_{2} \leq\|\mathrm{v}\|_{\mathrm{H}^{*}}\left|\int_{\mathrm{Q}}\left[\int_{0}^{\mathrm{t}}|\mathrm{A}|^{2} \mathrm{~d} \tau \int_{0}^{\mathrm{t}}|\mathrm{u}|^{2} \mathrm{~d} \tau\right] \mathrm{dQ}\right|^{1 / 2} \leq\left.\left.\|\mathrm{v}\|_{\mathrm{H}^{*}}\left|\frac{\mathrm{T}^{2}}{2} \int_{\Omega}\right| \mathrm{A}\right|^{2} \int_{0}^{\mathrm{T}}|\mathrm{u}|^{2} \mathrm{dtd} \Omega\right|^{1 / 2} \leq \mathrm{C}_{\mathrm{A}, \mathrm{T}}\|\mathrm{v}\|_{\mathrm{H}^{*}}\|\mathrm{u}\|_{\mathrm{H}},
$$

where $\mathrm{C}_{\mathrm{A}, \mathrm{T}}=$ const $>0$.

$$
\mathrm{I}_{3}=\left|\int_{\mathrm{Q}} \mathrm{vBudQ}\right| \leq 2 \mathrm{~V}^{2}\|\mathrm{v}\|_{\mathrm{L}_{2}(\mathrm{Q})}\left|\int_{\mathrm{Q}} \sum_{\mathrm{i}=1}^{2}\left(\frac{\partial \mathrm{u}}{\partial \mathrm{x}_{\mathrm{i}}}\right)^{2} \mathrm{dQ}\right|^{1 / 2} \leq \mathrm{c}_{\mathrm{V}}\|\mathrm{v}\|_{\mathrm{H}^{*}}\|\mathrm{u}\|_{\mathrm{H}}, \mathrm{c}_{\mathrm{V}}=\text { const }>0 .
$$

From (8)-(10) have

$$
\left|(\mathrm{Zu}, \mathrm{v})_{\mathrm{L}_{2}(\mathrm{Q})}\right| \leq \tilde{\mathrm{n}}_{1}\|\mathrm{v}\|_{\mathrm{H}^{*}}\|\mathrm{u}\|_{\mathrm{H}}
$$

Taking into account that

$$
\|\mathrm{Zu}\|_{\mathrm{H}^{*-}}=\sup _{\mathrm{v} \neq 0} \frac{\left|(\mathrm{Zu}, \mathrm{v})_{\mathrm{L}_{2}(\mathrm{Q})}\right|}{\|\mathrm{v}\|_{\mathrm{H}^{*}}}, \mathrm{v} \in \mathrm{H}^{*}
$$

and the density of the set $\mathrm{D}(\mathrm{Z})$ in space $\mathrm{H}$ and completeness of space $\mathrm{H}^{*-}$ (7).

Similarly (7) have

$$
\left\|\mathrm{Z}^{*} \mathrm{v}\right\|_{\mathrm{H}^{-}} \leq \mathrm{c}_{2}\|\mathrm{v}\|_{\mathrm{H}^{*}}, \quad \mathrm{c}_{2}=\text { const }>0
$$

for any $\mathrm{v} \in \mathrm{H}^{*}$

It is proposed to consider the solution of task (1)-(3) in the following sense.

The generalized solution of the problem (1)-(3) is a function $u(x, t) \in H$ such that there is a sequence of functions $u_{3}(x, t) \in D(Z), i=1,2, \ldots$, that satisfy the conditions (2), (3) and

$$
\left\|\mathrm{Z} \mathrm{u}_{\mathrm{i}}-\mathrm{f}\right\|_{\mathrm{H}^{--}} \rightarrow 0,\left\|\mathrm{u}_{\mathrm{i}}-\mathrm{u}\right\|_{\mathrm{H}} \rightarrow 0, \mathrm{i} \rightarrow \infty
$$

The generalized solution of the problem (1)-(3) is a function $u(x, t) \in L_{2}(Q)$ such that there is a sequence of functions

$$
\mathrm{u}_{3}(\mathrm{x}, \mathrm{t}) \in \mathrm{D}(\mathrm{Z}), \mathrm{i}=1,2, \ldots,
$$

that satisfy the conditions (2), (3) and

$$
\left\|\mathrm{Zu}_{\mathrm{i}}-\mathrm{f}\right\|_{\mathrm{H}^{*-}} \rightarrow 0, \quad\left\|\mathrm{u}_{\mathrm{i}}-\mathrm{u}\right\|_{\mathrm{L}_{2}(\mathrm{Q})} \rightarrow 0, \mathrm{i} \rightarrow \infty
$$

Then for any $f \in L_{2}(Q)$ there exists the unique solution of problem (1)-(3) as function $u \in H$. Let's prove this statement. For this w let's consider the functional

$$
l_{\mathrm{f}}(\mathrm{v}) \equiv(\mathrm{v}, \mathrm{f})_{\mathrm{L}_{2}(\mathrm{Q})} .
$$

From the form of the function $f$ let's have $\mathrm{f} \leq \hat{\mathrm{c}}\|\mathrm{v}(0, \mathrm{x})\|_{\mathrm{L}_{2}(\Omega)}$ and therefore 


$$
l_{\mathrm{f}}(\mathrm{v}) \leq\|\mathrm{v}\|_{L_{2}(\mathrm{Q})}\|\mathrm{f}\|_{\mathrm{L}_{2}(\mathrm{Q})} \leq \mathrm{c}\|\mathrm{v}\|_{\mathrm{L}_{2}(\mathrm{Q})} \leq \overline{\mathrm{c}}\|\mathrm{v}\|_{\mathrm{H}^{*}} .
$$

The functional $l_{\mathrm{f}}(\mathrm{v})$ is linear and continuous therefore it follows that there exists the unique $\mathrm{f} \in \mathrm{H}^{*-}$ such that $\mathrm{l}(\mathrm{v})=\langle\mathrm{v}, \mathrm{f}\rangle_{\mathrm{Q}}$ for any $\mathrm{v} \in \mathrm{H}^{*}$. The functional $\mathrm{l}(\mathrm{v})$ on functions $\mathrm{v} \in \mathrm{D}\left(\mathrm{Z}^{*}\right)$ is

$$
1(\mathrm{v})=\langle\mathrm{v}, \mathrm{f}\rangle_{\mathrm{Q}}=\left(\mathrm{Z}^{*} \mathrm{v}, \mathrm{u}\right)_{\mathrm{L}_{2}(\mathrm{Q})}
$$

Since $D(Z)$ is dense in $H$, then there exists a sequence $u_{3} \in D(Z), i=1,2, \ldots$, such that $\left\|\mathrm{u}_{\mathrm{i}}-\mathrm{u}\right\|_{\mathrm{I}} \rightarrow 0, \mathrm{i} \rightarrow \infty$. Using the fundamental sequence of $\mathrm{u}_{3}, \mathrm{i}=1,2, \ldots$, where

$$
\left\|u_{i}-u_{j}\right\|_{H} \rightarrow 0, \quad i, j \rightarrow \infty,
$$

and taking into account the linearity of the operator $\mathrm{Z}$ and inequality (7), obtain that

$$
\left\|\mathrm{Zu}_{\mathrm{i}}-\mathrm{Zu}_{\mathrm{j}}\right\|_{\mathrm{i}^{*-}}=\left\|\mathrm{Z}\left(\mathrm{u}_{\mathrm{i}}-\mathrm{u}_{\mathrm{j}}\right)\right\|_{\mathrm{i} \cdot-} \leq \mathrm{c}_{1}\left\|\mathrm{u}_{\mathrm{i}}-\mathrm{u}_{\mathrm{j}}\right\|_{\mathrm{i}} .
$$

Hence, bearing in mind the completeness of $\mathrm{H}^{*-}$, there exists $\hat{\mathrm{f}} \in \mathrm{H}^{*-}$ such that

$$
\left\|Z u_{i}-\hat{f}\right\|_{\hat{I}^{*}} \rightarrow 0, i \rightarrow \infty \text {. }
$$

And as for any $\mathrm{v} \in \mathrm{D}\left(\mathrm{Z}^{*}\right)$

$$
\left\langle\mathrm{v}, \mathrm{Z} \mathrm{u}_{\mathrm{i}}-\hat{\mathrm{f}}\right\rangle_{\mathrm{Q}} \leq\|\mathrm{v}\|_{\mathrm{H}^{*}}\left\|\mathrm{Zu_{ \textrm {i } }}-\hat{\mathrm{f}}\right\|
$$

then

$$
\left\langle\mathrm{v}, \mathrm{Zu} \mathrm{u}_{\mathrm{i}}-\hat{\mathrm{f}}\right\rangle_{\mathrm{Q}} \rightarrow 0
$$

and

$$
\left|\left(\mathrm{Zu}_{\mathrm{i}}, \mathrm{v}\right)_{\mathrm{L}_{2}(\mathrm{Q})}-(\mathrm{f}, \mathrm{v})_{\mathrm{L}_{2}(\mathrm{Q})}\right| \leq\left\|\mathrm{u}_{\mathrm{i}}-\mathrm{u}\right\|_{\mathrm{H}}\left\|\mathrm{Z}^{*} \mathrm{v}\right\|_{\mathrm{H}^{*-}} \rightarrow 0, \mathrm{i} \rightarrow \infty .
$$

So true

$$
\left|\langle\mathrm{v}, \mathrm{f}-\hat{\mathrm{f}}\rangle_{\mathrm{Q}}\right| \leq\left|\left\langle\mathrm{v}, \mathrm{Zu_{i }}-\mathrm{f}\right\rangle_{\mathrm{Q}}\right|+\left|\left\langle\mathrm{v}, \mathrm{Zu_{i }}-\hat{\mathrm{f}}\right\rangle_{\mathrm{Q}}\right| \rightarrow 0, \mathrm{i} \rightarrow \infty .
$$

Then for any $\mathrm{v} \in \mathrm{D}\left(\mathrm{Z}^{*}\right)$ have

$$
\langle v, f-\hat{f}\rangle_{Q}=0
$$

And since the set of functions $v \in D\left(Z^{*}\right)$ is dense in $H^{*}$, the functional $\langle v, f-\hat{f}\rangle_{Q}$ is linear and bounded, then $\|\mathrm{f}-\hat{\mathrm{f}}\|_{\mathrm{H}^{*-}} \rightarrow 0$. Taking into account (7) have

$$
\left\|\mathrm{Zu}_{\mathrm{i}}-\mathrm{f}\right\|_{\mathrm{H}^{--}} \rightarrow 0, \mathrm{i} \rightarrow \infty
$$

and therefore function $\mathrm{u} \in \mathrm{H}$ is a unique solution of the problem (1)-(3).

Further, for any $f \in \mathrm{H}^{*-}(\mathrm{Q})$ there exists the unique solution of (1)-(3) as function $\mathrm{u} \in \mathrm{L}_{2}(\mathrm{Q})$.

Let's prove this statement. The space $\mathrm{L}_{2}(\mathrm{Q})$ is dense in $\mathrm{H}^{*-}(\mathrm{Q})$, so for any $\mathrm{f} \in \mathrm{H}^{*-}$ there exists a sequence

$$
\mathrm{f}_{3} \in \mathrm{L}_{2}(\mathrm{Q}), \mathrm{i}=1,2, \ldots, \quad\left\|\mathrm{f}_{\mathrm{i}}-\mathrm{f}\right\|_{\hat{I}^{*}{ }^{-}} \rightarrow 0, \mathrm{i} \rightarrow \infty .
$$


And since for any $\mathrm{f}_{3} \in \mathrm{L}_{2}(\mathrm{Q})$ there exists a unique solution $\mathrm{u}_{\mathrm{i}} \in \mathrm{H}(\mathrm{Q})$ for the task (1)-(3) (namely, there exists a subsequence

$$
\left\{\mathrm{u}_{\mathrm{s}_{\mathrm{j}}}\right\}_{\mathrm{j}=1}^{\infty} \in \mathrm{D}(\mathrm{Z})
$$

such that $\left.\left\|\mathrm{Zu}_{\mathrm{i}_{\mathrm{j}}}-\mathrm{f}_{\mathrm{i}}\right\|_{\mathrm{H}^{\mathrm{t}^{-}}} \rightarrow 0,\left\|\mathrm{u}_{\mathrm{i}_{\mathrm{j}}}-\mathrm{u}_{\mathrm{i}}\right\|_{\mathrm{H}} \rightarrow 0, \mathrm{j} \rightarrow \infty\right)$ and

$$
\left\|\mathrm{u}_{\mathrm{i}_{\mathrm{j}}}-\mathrm{u}_{\mathrm{i}}\right\|_{\mathrm{H}} \geq \mathrm{c}\left\|\mathrm{u}_{\mathrm{i}_{\mathrm{j}}}-\mathrm{u}_{\mathrm{i}}\right\|_{\mathrm{L}_{2}(Q)},
$$

then for limit at $\mathrm{i} \rightarrow \infty, \quad \mathrm{j} \rightarrow \infty$ have the existence of unique solution of (1)-(3) as a function $\mathrm{u} \in \mathrm{L}_{2}(\mathrm{Q})$.

\section{2. Basic properties of differential operators}

Let's write equation (1) as $\mathrm{Zu} \equiv \mathrm{u}_{\mathrm{t}}+\mathrm{Au}+\mathrm{Bu}=\mathrm{f}$ and consider $(\mathrm{Zu}, \mathrm{u})_{\mathrm{L}_{2}(\mathrm{Q})}$. Then

$$
\left(\mathrm{u}_{\mathrm{t}}, \mathrm{u}\right)_{\mathrm{L}_{2}(\mathrm{Q})}=\frac{1}{2} \int_{\mathrm{Q}} \frac{\partial}{\partial \mathrm{t}}\left(\mathrm{u}^{2}\right) \mathrm{dQ}=\frac{1}{2} \int_{\Omega} \mathrm{u}^{2}(\mathrm{~T}) \mathrm{d} \Omega=\frac{1}{2}\|\mathrm{u}(\mathrm{T})\|_{\mathrm{L}_{2}(\Omega)}^{2},
$$

$(\mathrm{Bu}, \mathrm{u})_{\mathrm{L}_{2}(\mathrm{Q})} \leq\|\mathrm{u}\|_{\mathrm{L}_{2}(\mathrm{Q})}\left(\int_{\mathrm{Q}}\left(\mathrm{V} \sum_{\mathrm{i}=1}^{2} \frac{\partial \mathrm{u}}{\partial \mathrm{x}_{\mathrm{i}}}\right)^{2} \mathrm{dQ}\right)^{1 / 2} \leq \sqrt{2} \mathrm{~V}\|\mathrm{u}\|_{\mathrm{L}_{2}(\mathrm{Q})}\|\mathrm{u}\|_{\mathrm{H}} \leq \tilde{\mathrm{c}}(\mathrm{V})\|\mathrm{u}\|_{\mathrm{H}}^{2}, \tilde{\mathrm{c}}(\mathrm{V})=$ const $>0$

and

$$
\begin{gathered}
(\mathrm{Bu}, \mathrm{u})_{\Omega} \leq \sqrt{2} \mathrm{~V}\|\mathrm{u}\|_{\mathrm{L}_{2}(\Omega)}\left\|\sum_{\mathrm{i}=1}^{2} \frac{\partial \mathrm{u}}{\partial \mathrm{x}_{\mathrm{i}}}\right\|_{\mathrm{L}_{2}(\Omega)} \leq \overline{\mathrm{c}}(\mathrm{V})\|\mathrm{u}\|_{\mathrm{W}_{2}^{1}(\Omega)}^{2}, \\
\overline{\mathrm{c}}(\mathrm{V})=\text { const }>0
\end{gathered}
$$

for any fixed $\mathrm{t} \in(0, \mathrm{~T})\left(\mathrm{W}_{2}^{1}(\Omega)-\right.$ the space with norm $\|\mathrm{u}\|_{\mathrm{W}_{2}^{1}(\Omega)}=\int_{\Omega}\left(\mathrm{u}^{2}+\sum_{\mathrm{i}=1}^{2}\left(\frac{\partial \mathrm{u}}{\partial \mathrm{x}_{\mathrm{i}}}\right)^{2} \mathrm{~d} \Omega\right)$.

For operator A:

$$
(A u, u)_{L_{2}(Q)}=\int_{Q} u\left(-\sum_{i=1}^{2}\left(\frac{\partial D}{\partial x_{i}} \frac{\partial u}{\partial x_{i}}+D(x) \frac{\partial^{2} u}{\partial x_{i}^{2}}\right)\right) d Q=I_{1}+I_{2},
$$

where

$$
\begin{gathered}
\mathrm{I}_{1} \leq\|\mathrm{u}\|_{\mathrm{L}_{2}(\mathrm{Q})}\left(\iint_{\mathrm{Q}}\left(\sum_{\mathrm{i}=1}^{2}\left(\frac{\partial \mathrm{D}}{\partial \mathrm{x}_{\mathrm{i}}}\right)^{2} \sum_{\mathrm{i}=1}^{2}\left(\frac{\partial \mathrm{u}}{\partial \mathrm{x}_{\mathrm{i}}}\right)^{2}\right) \mathrm{dQ}\right)^{1 / 2} \leq \mathrm{c}_{\max _{\mathrm{D}}}\|\mathrm{u}\|_{\mathrm{L}_{2}(\mathrm{Q})}\|\mathrm{u}\|_{\mathrm{H}} \leq \mathrm{c}_{\mathrm{D}}\|\mathrm{u}\|_{\mathrm{H}}^{2}, \quad \mathrm{c}_{\mathrm{D}}=\text { const }>0, \\
\mathrm{I}_{2} \leq\|\mathrm{u}\|_{\mathrm{L}_{2}(\mathrm{Q})}\left(\int_{\mathrm{Q}} 2 \mathrm{D}^{2}\left(\sum_{\mathrm{i}=1}^{2}\left(\frac{\partial^{2} \mathrm{u}}{\partial \mathrm{x}_{\mathrm{i}}^{2}}\right)^{2}\right) \mathrm{dQ}\right)^{1 / 2} \leq \mathrm{c}_{\mathrm{D} \max }\|\mathrm{u}\|_{\mathrm{L}_{2}(\mathrm{Q})}\|\mathrm{u}\|_{\mathrm{H}} \leq \overline{\mathrm{c}}_{\mathrm{D}}\|\mathrm{u}\|_{\mathrm{H}}^{2}, \quad \overline{\mathrm{c}}_{\mathrm{D}}=\text { const }>0 .
\end{gathered}
$$

From (11)-(14)

$$
(\mathrm{Zu}, \mathrm{u})_{\mathrm{L}_{2}(\mathrm{Q})} \leq \mathrm{c}\|\mathrm{u}\|_{\mathrm{H}}^{2}, \quad \mathrm{c}=\text { const }>0 .
$$

In addition, there are operator B following estimates: 


$$
\|\mathrm{Bu}\|_{\Omega}^{2} \leq 2 \overline{\mathrm{c}}{ }^{2}\|\mathrm{u}\|_{\mathrm{W}_{2}^{1}(\Omega)}^{2}
$$

and

$$
\|\mathrm{Bu}\|_{\mathrm{Q}}^{2} \leq 2 \sum_{\mathrm{i}=1}^{2} \int_{\mathrm{Q}} \mathrm{V}^{2}\left(\frac{\partial \mathrm{u}}{\partial \mathrm{x}_{\mathrm{i}}}\right)^{2} \mathrm{dQ} \leq 2 \overline{\mathrm{c}} \mathrm{V}^{2}\|\mathrm{u}\|_{\mathrm{H}}^{2}, \overline{\mathrm{c}}=\text { const }>0 .
$$

Next, let's proceed to the expression $(\mathrm{Zu}, \mathrm{u})_{\mathrm{L}_{2}(\mathrm{Q})}=(\mathrm{f}, \mathrm{u})_{\mathrm{L}_{2}(\mathrm{Q})}$. It is true:

$$
\begin{aligned}
(\mathrm{Zu}, \mathrm{u})_{\mathrm{L}_{2}(\mathrm{Q})} & =\frac{1}{2} \frac{\partial}{\partial \mathrm{t}}\|\mathrm{u}\|_{\mathrm{L}_{2}(\mathrm{Q})}^{2}+(\mathrm{Au}, \mathrm{u})_{\mathrm{L}_{2}(\mathrm{Q})}+(\mathrm{Bu}, \mathrm{u})_{\mathrm{L}_{2}(\mathrm{Q})} \leq\|\mathrm{f}\|_{\mathrm{L}_{2}(\mathrm{Q})}\|\mathrm{u}\|_{\mathrm{L}_{2}(\mathrm{Q})}, \\
(\mathrm{Zu}, \mathrm{u})_{\mathrm{L}_{2}(\mathrm{Q})} & \leq\|\mathrm{u}\|_{\mathrm{L}_{2}(\mathrm{Q})} \frac{\partial}{\partial \mathrm{t}}\|\mathrm{u}\|_{\mathrm{L}_{2}(\mathrm{Q})}+\|\mathrm{u}\|_{\mathrm{L}_{2}(\mathrm{Q})}\|\mathrm{Au}\|_{\mathrm{L}_{2}(\mathrm{Q})}+\|\mathrm{u}\|_{\mathrm{L}_{2}(\mathrm{Q})}\|\mathrm{Bu}\|_{\mathrm{L}_{2}(\mathrm{Q})},
\end{aligned}
$$

where considering that $u \in D(Z)$ have

$$
\left(\mathrm{u}, \mathrm{u}_{\mathrm{t}}\right)_{\mathrm{L}_{2}(\mathrm{Q})}=\frac{1}{2} \frac{\partial}{\partial \mathrm{t}}\|\mathrm{u}\|_{\mathrm{L}_{2}(\mathrm{Q})}^{2}=\|\mathrm{u}\|_{\mathrm{L}_{2}(\mathrm{Q})} \frac{\partial}{\partial \mathrm{t}}\|\mathrm{u}\|_{\mathrm{L}_{2}(\mathrm{Q})}, \quad\left(\mathrm{u}, \mathrm{u}_{\mathrm{t}}\right)_{\mathrm{L}_{2}(\mathrm{Q})}=\frac{1}{2}\|\mathrm{u}(\mathrm{T})\|_{\mathrm{L}_{2}(\Omega)}^{2}
$$

and gives that

$$
\frac{\partial}{\partial \mathrm{t}}\|\mathrm{u}\|_{\mathrm{L}_{2}(\mathrm{Q})} \geq 0 \text { and } \frac{\partial}{\partial \mathrm{t}}\left(\int_{\mathrm{Q}} \mathrm{u}^{2} \mathrm{dQ}\right)^{1 / 2} \leq\|\mathrm{u}\|_{\mathrm{H}}
$$

Then,

$$
(\mathrm{Bu}, \mathrm{u})_{\mathrm{L}_{2}(\mathrm{Q})}=\mathrm{V}\|\mathrm{u}\|_{\mathrm{L}_{2}(\mathrm{Q})} \sum_{\mathrm{i}=1}^{2} \frac{\partial}{\partial \mathrm{x}_{\mathrm{i}}}\|\mathrm{u}\|_{\mathrm{L}_{2}(\mathrm{Q})}
$$

and from the above obtained

$$
\frac{\partial}{\partial \mathrm{t}}\|\mathrm{u}\|_{\mathrm{L}_{2}(\mathrm{Q})}+\mathrm{V} \sum_{\mathrm{i}=1}^{2} \frac{\partial}{\partial \mathrm{x}_{\mathrm{i}}}\|\mathrm{u}\|_{\mathrm{L}_{2}(\mathrm{Q})} \leq\|\mathrm{f}\|_{\mathrm{L}_{2}(\mathrm{Q})}+\mathrm{C}_{\mathrm{D}}\|\mathrm{u}\|_{\mathrm{H}}, \quad \mathrm{C}_{\mathrm{D}}=\text { const }>0
$$

\section{3. Difference analogues of differential operators and their properties}

The difference analogue was constructed for the considered problem of migration of a pollutant in the soil. To do this, a difference grid for both variables in $\Omega_{h}$ with a steady step $h_{1}, h_{2}$ was introduced. Let $\omega_{\mathrm{h}}$ be the set of internal nodes of the grid

$$
\omega=\left\{x \mid x=\left(x_{1}, x_{2}\right), x_{1}=i_{1} h_{1}, x_{2}=i_{2} h_{2}, i_{1}=\overline{1, N_{1}-1}, i_{2}=\overline{1, N_{2}-1}, N_{1} h_{1}=L_{1}, i_{2} h_{2}=L_{2}\right\},
$$

and let $\partial \omega$ be the set of external nodes. The difference solution of the task at the time $t$ is denoted by

$$
y(x, t), x \in \omega \cup \partial \omega, t>0 .
$$

For the grid functions satisfying the conditions (2), (3) on $\partial \omega$, the space $\overline{\mathrm{H}}$ is defined, in which the scalar product and norm are:

$$
(\mathrm{y}, \mathrm{v})_{\overline{\mathrm{H}}}=\sum_{\mathrm{x} \in \omega} \mathrm{y} v \mathrm{~h}_{1} \mathrm{~h}_{2},\|\mathrm{y}\|_{\overline{\mathrm{H}}}=\sqrt{(\mathrm{y}, \mathrm{y})_{\overline{\mathrm{H}}}}=\left(\sum_{\mathrm{x} \in \omega} \mathrm{y}_{\mathrm{j}_{1} \mathrm{j}_{2}}^{2} \mathrm{~h}_{1} \mathrm{~h}_{2}\right)^{1 / 2} .
$$


To the operator $A$ on the set of functions $y \in \overline{\mathrm{H}}$ let's put into conformity the difference operator

$$
A=-\sum_{i=1}^{2}\left(D y_{x_{i}}\right)_{\bar{x}_{i}}, y_{x_{i}}=\frac{y\left(x_{i}+h_{i}\right)-y\left(x_{i}\right)}{h_{i}}, y_{\bar{x}_{i}}=\frac{y\left(x_{i}\right)-y\left(x_{i}-h_{i}\right)}{h_{i}} .
$$

To the operator $\mathrm{B}$ on the set of functions $\mathrm{y} \in \overline{\mathrm{H}}$ let's e put into conformity the difference operator

$$
\mathrm{B}=\sum_{\mathrm{i}=1}^{2} \mathrm{Vy}_{\hat{\mathrm{x}}_{\mathrm{i}}},
$$

where $\mathrm{y}_{\hat{\mathrm{x}}_{\mathrm{i}}}=\left(\mathrm{y}_{\mathrm{x}_{\mathrm{i}}}+\mathrm{y}_{\overline{\mathrm{x}}_{\mathrm{i}}}\right) / 2$.

Let's consider the basic properties of difference operators in space $\bar{H}$. First, will take

$$
(B y, v)_{\overline{\mathrm{H}}}=\sum_{\mathrm{i}=1}^{2} \sum_{\mathrm{x} \in \boldsymbol{\omega}} \mathrm{Vy}_{\hat{\mathrm{x}}_{\mathrm{i}}} v(\mathrm{x}) \mathrm{h}_{1} \mathrm{~h}_{2} .
$$

On the set of grid functions in the internal nodes $\omega_{\mathrm{h}}$ :

$$
\sum_{\mathrm{x} \in \omega} \mathrm{Vy}_{\hat{\mathrm{x}}_{1}} v(\mathrm{x}) \mathrm{h}_{1} \mathrm{~h}_{2}=\sum_{\mathrm{x} \in \omega} \mathrm{V}(\mathrm{y} v)_{\hat{\mathrm{x}}_{1}} \mathrm{~h}_{1} \mathrm{~h}_{2}-\sum_{\mathrm{x} \in \omega} V \mathrm{~V} \mathrm{v}_{\hat{\mathrm{x}}_{1}} \mathrm{~h}_{1} \mathrm{~h}_{2} .
$$

The same holds for the second variable. Then for any $y \in \overline{\mathrm{H}}$ is executed

$$
(\mathrm{By}, \mathrm{y})_{\overline{\mathrm{H}}} \leq \mathrm{c}\|\mathrm{y}\|_{\overline{\mathrm{H}}}^{2}, \mathrm{c}=\text { const }>0 .
$$

The proof.

$$
(\text { By, y })_{\overline{\mathrm{H}}}=\sum_{\mathrm{i}=1}^{2} \sum_{\mathrm{x} \in \omega} \mathrm{Vy}_{\hat{\mathrm{x}}_{\mathrm{i}}} \mathrm{yh}_{1} \mathrm{~h}_{2} \leq \sum_{\mathrm{i}=1}^{2} \sum_{\mathrm{x} \in \omega} \frac{\mathrm{V}}{4 \mathrm{~h}_{\mathrm{i}}} \mathrm{y}^{2}\left(\mathrm{x}_{\mathrm{i}}+\mathrm{h}_{\mathrm{i}}\right) \mathrm{h}_{1} \mathrm{~h}_{2} \leq \frac{\mathrm{V}}{4 \mathrm{~h}_{\min }}\|\mathrm{y}\|_{\overline{\mathrm{H}}}^{2}
$$

and for $\mathrm{h}_{\min }=\min \left\{\mathrm{h}_{1}, \mathrm{~h}_{2}\right\}$ the accuracy of approximation is $\hat{\mathrm{I}}\left(\mathrm{h}_{3}^{2}\right)$. The fidelity of (19) follows from (20).

Let's show that for any $y \in \overline{\mathrm{H}}$ is true

$$
\begin{gathered}
\|B y\|_{\overline{\mathrm{H}}}^{2} \leq \hat{\mathrm{c}}\|\mathrm{y}\|_{\overline{\mathrm{H}}}^{2}, \hat{\mathrm{c}}=\text { const }>0 . \\
\|\mathrm{By}\|_{\overline{\mathrm{H}}}^{2} \leq 2 \mathrm{~V}^{2} \sum_{\mathrm{i}=1}^{2} \sum_{\mathrm{x} \in \omega} \frac{1}{4}\left[\mathrm{y}_{\mathrm{x}_{\mathrm{i}}}+\mathrm{y}_{\overline{\mathrm{x}}_{\mathrm{i}}}\right]^{2} \mathrm{~h}_{1} \mathrm{~h}_{2} \leq 8 \mathrm{~V}^{2} \sum_{\mathrm{i}=1}^{2} \sum_{\mathrm{x} \in \omega} \frac{1}{\mathrm{~h}_{\mathrm{i}}^{2}} \mathrm{y}_{\mathrm{jin}_{\mathrm{j}}}^{2} \mathrm{~h}_{1} \mathrm{~h}_{2} \leq \frac{8 \mathrm{~V}^{2}}{\mathrm{~h}^{2}{ }_{\text {min }}}\|\mathrm{y}\|_{\overline{\mathrm{H}}}^{2} .
\end{gathered}
$$

From (21) wit is possible to obtain the required.

Moreover, for any $\mathrm{y} \in \overline{\mathrm{H}}$

$$
(\text { Ay, },)_{\overline{\mathrm{H}}} \leq \mathrm{c}\|\mathrm{y}\|_{\overline{\mathrm{H}}}^{2}, \mathrm{c}=\text { const }>0 .
$$

The proof.

$$
(A y, y)_{\overline{\mathrm{H}}}=-\sum_{\mathrm{i}=1}^{2} \sum_{\mathrm{x} \in \omega}\left(\mathrm{a}_{\mathrm{j}_{\mathrm{i}}+1} \frac{\mathrm{y}_{\mathrm{j}_{\mathrm{i}}+1}-\mathrm{y}_{\mathrm{j}_{\mathrm{i}}}}{\mathrm{h}_{\mathrm{i}}}-\mathrm{a}_{\mathrm{j}_{\mathrm{i}}} \frac{\mathrm{y}_{\mathrm{j}_{\mathrm{i}}}-\mathrm{y}_{\mathrm{j}_{\mathrm{i}}-1}}{\mathrm{~h}_{\mathrm{i}}}\right) \mathrm{y}_{\mathrm{j}_{\mathrm{i}, 2} \mathrm{j}_{2}} \mathrm{~h}_{1} \mathrm{~h}_{2} .
$$

The coefficients $\mathrm{a}_{\mathrm{j}_{1} \mathrm{j}_{2}}$ are chosen from the conditions of the second order of approximation from the decomposition of 


$$
\frac{\mathrm{a}_{\mathrm{j}_{\mathrm{i}}}+\mathrm{a}_{\mathrm{j}_{\mathrm{i}}+1}}{2}=\mathrm{D}_{\mathrm{j}_{\mathrm{i}}}+\mathrm{O}\left(\mathrm{h}_{\mathrm{i}}^{2}\right), \frac{\mathrm{a}_{\mathrm{j}_{\mathrm{i}}+1}-\mathrm{a}_{\mathrm{j}_{\mathrm{i}}}}{\mathrm{h}_{\mathrm{i}}}=\mathrm{D}_{\mathrm{x}_{\mathrm{i}}}+\mathrm{O}\left(\mathrm{h}_{\mathrm{i}}^{2}\right),
$$

i. e.

$$
\mathrm{a}_{\mathrm{j}_{\mathrm{i}}}=\frac{\mathrm{D}_{\mathrm{j}_{\mathrm{i}}}+\mathrm{D}_{\mathrm{j}_{\mathrm{i}}+1}}{2}, \quad \mathrm{D}_{\mathrm{j}_{\mathrm{i}}}=\left.\mathrm{D}\left(\mathrm{x}_{1}, \mathrm{x}_{2}\right)\right|_{\mathrm{x}_{\mathrm{j}}=\mathrm{j}_{\mathrm{i}} \mathrm{h}_{\mathrm{i}}}, \mathrm{j}_{\mathrm{i}}=\overline{1, \mathrm{~N}_{\mathrm{i}}-1}, \mathrm{i}=\overline{1,2}
$$

Therefore

$$
(\text { Ay,y })_{\overline{\mathrm{H}}} \leq \sum_{\mathrm{i}=1}^{2} \sum_{\mathrm{x} \in \omega} \frac{1}{\mathrm{~h}_{\mathrm{i}}}\left(\mathrm{a}_{\mathrm{j}_{\mathrm{i}}+1}+\mathrm{a}_{\mathrm{j}_{\mathrm{i}}}\right) \mathrm{y}_{\mathrm{j}_{1} \mathrm{j}_{2}}^{2} \mathrm{~h}_{1} \mathrm{~h}_{2} \leq \mathrm{c}\|\mathrm{y}\|_{\overline{\mathrm{H}}}^{2}, \mathrm{c}=\text { const }>0,
$$

where

$$
\begin{gathered}
\sum_{\mathrm{i}=1}^{2} \sum_{\mathrm{x} \in \omega} \frac{1}{\mathrm{~h}_{\mathrm{i}}}\left(\mathrm{a}_{\mathrm{j}_{\mathrm{i}}+1}+\mathrm{a}_{\mathrm{j}_{\mathrm{i}}}\right) \leq \sum_{\mathrm{i}=1}^{2} \frac{2\left(\mathrm{~N}_{\mathrm{i}}-1\right) \mathrm{D}_{\max }}{\mathrm{h}_{\mathrm{i}}} \leq \frac{4(\mathrm{~N}-1) \mathrm{D}_{\max }}{\mathrm{h}_{\min }}, \\
\mathrm{N}=\max \left\{\mathrm{N}_{1}, \mathrm{~N}_{2}\right\}, \quad \mathrm{D}_{\max }=\max _{\mathrm{j}_{\alpha}}\left\{\mathrm{D}_{\mathrm{j}_{\alpha}}\right\}, \alpha=1,2 .
\end{gathered}
$$

From (23) the fidelity of inequality (22) follows.

Let's show that for any $\mathrm{y} \in \overline{\mathrm{H}}$ is true

$$
\begin{aligned}
& \|\mathrm{Ay}\|_{\overline{\mathrm{H}}}^{2} \leq \tilde{\mathrm{c}}\|\mathrm{y}\|_{\overline{\mathrm{H}}}^{2}, \tilde{\mathrm{c}}=\text { const }>0 . \\
& \|A y\|_{\overline{\mathrm{H}}}^{2} \leq 4 \sum_{\mathrm{x} \in \omega}\left(\sum_{\mathrm{i}=1}^{2}\left(\left(\mathrm{a}_{\mathrm{j}_{\mathrm{i}}+1} \mathrm{y}_{\mathrm{x}_{\mathrm{i}}}\right)^{2}+\left(\mathrm{a}_{\mathrm{j}_{\mathrm{i}}} \mathrm{y}_{\overline{\mathrm{x}}_{\mathrm{i}}}\right)^{2}\right)\right) \mathrm{h}_{1} \mathrm{~h}_{2} \leq 64 \sum_{\mathrm{x} \in \omega}\left(\sum_{\mathrm{i}=1}^{2} \frac{1}{\mathrm{~h}_{\mathrm{i}}^{2}}\left(\mathrm{D}_{\mathrm{j}_{\mathrm{i}}}^{2} \mathrm{y}_{\mathrm{j}_{\mathrm{i}} \mathrm{j}_{2}}^{2}\right)\right) \mathrm{h}_{1} \mathrm{~h}_{2} \leq 64 \frac{\mathrm{D}_{\max }^{2}}{\mathrm{~h}_{\min }^{2}}\|\mathrm{y}\|_{\overline{\mathrm{H}}}^{2} .
\end{aligned}
$$

From (24) it is possible to obtain the required.

\section{4. Difference scheme for the model}

As a result of discretization let's obtain the operator-difference scheme, which is the difference analog of differential problem with the order of approximation $\mathrm{O}\left(\tau+\mathrm{h}^{2}\right)$.

Let $\mathrm{y}^{(\mathrm{k})}$ be a difference solution at the time $t_{\mathrm{k}}=\mathrm{k} \tau$, where $\tau>0$. The two-layer difference scheme with weights has the form:

$$
\begin{gathered}
\frac{\mathrm{y}^{(\mathrm{k}+1)}-\mathrm{y}^{(\mathrm{k})}}{\tau}+\mathrm{A}\left[\sigma_{1} \mathrm{y}^{(\mathrm{k}+1)}+\left(1-\sigma_{1}\right) \mathrm{y}^{(\mathrm{k})}\right]+\mathrm{B}\left[\sigma_{2} \mathrm{y}^{(\mathrm{k}+1)}+\left(1-\sigma_{2}\right) \mathrm{y}^{(\mathrm{k})}\right]=\phi^{\mathrm{k}} \\
\mathrm{x} \in \omega, \mathrm{k}=0,1, \ldots
\end{gathered}
$$

with initial conditions

$$
\mathrm{y}^{(0)}(\mathrm{x})=0, \mathrm{x} \in \omega
$$

Let's write the scheme in canonical form for $\sigma_{1}=\sigma_{2}=\sigma$

$$
\begin{gathered}
\underline{\mathrm{B}} \frac{\mathrm{y}^{(\mathrm{k}+1)}-\mathrm{y}^{(\mathrm{k})}}{\tau}+\underline{\mathrm{Ay}}^{(\mathrm{k})}=\phi^{(\mathrm{k})}, \mathrm{x} \in \omega, \mathrm{k}=0,1, \ldots, \\
\underline{\mathrm{B}}=\mathrm{E}+\sigma \tau \underline{\mathrm{A}}, \underline{\mathrm{A}}=\mathrm{A}+\mathrm{B} .
\end{gathered}
$$

The operators $\underline{\mathrm{A}}, \underline{\mathrm{B}}$ are non-self-adjoint and non-sign-valued. 
Let's prove that the inhomogeneous difference scheme is stable:

- the condition

$$
\frac{\mathrm{V}}{2}>\mathrm{a}_{\mathrm{j}_{1} \mathrm{j}_{2}}, \mathrm{j}_{\mathrm{i}}=\overline{1, \mathrm{~N}_{\mathrm{i}}}, \mathrm{i}=\overline{1,2}
$$

for

$$
\tau\left(\frac{1}{\mathrm{~h}_{1}}+\frac{1}{\mathrm{~h}_{2}}\right)<\left(\sigma\left(\mathrm{V}-2 \mathrm{D}_{\mathrm{j}_{1} \mathrm{j}_{2}}\right)\right)^{-1}
$$

- under the condition

$$
\frac{\mathrm{V}}{2}<\mathrm{a}_{\mathrm{j}_{1} \mathrm{j}_{2}}, \mathrm{j}_{\mathrm{i}}=\overline{1, \mathrm{~N}_{\mathrm{i}}}, \mathrm{i}=\overline{1,2}
$$

for

$$
\tau\left(\frac{1}{\mathrm{~h}_{1}}+\frac{1}{\mathrm{~h}_{2}}\right)<\left(2 \sigma\left(\mathrm{D}_{\max }-0,25 \mathrm{~V}\right)\right)^{-1}
$$

The proof.

$$
\underline{B}=\mathrm{E}+\sigma \tau \underline{\mathrm{A}}, \underline{\mathrm{A}}=\mathrm{A}+\mathrm{B}, \underline{\mathrm{A}} \neq \underline{\mathrm{A}}^{*}
$$

and

$$
\|\underline{\mathrm{Ay}}\|_{\overline{\mathrm{H}}}^{2} \leq 2\left(\|\mathrm{Ay}\|_{\overline{\mathrm{H}}}^{2}+\|\mathrm{By}\|_{\overline{\mathrm{H}}}^{2}\right) \leq 2\left(\frac{64 \mathrm{D}_{\max }}{\mathrm{h}_{\text {min }}^{2}}+\frac{8 \mathrm{~V}^{2}}{\mathrm{~h}_{\text {min }}^{2}}\right)\|\mathrm{y}\|_{\overline{\mathrm{H}}}^{2}=\mathrm{M}_{1}\|\mathrm{y}\|_{\overline{\mathrm{H}}}^{2}, \mathrm{M}_{1}=\text { const }>0 .
$$

And consequently

$$
\|\underline{\mathrm{By}}\|_{\overline{\mathrm{H}}}^{2} \leq \mathrm{M}\|\mathrm{y}\|_{\overline{\mathrm{H}}}^{2}, \quad \mathrm{M}=\mathrm{const}>0 .
$$

One of the conditions of stability is $\underline{B}>0$. Let's consider what $\sigma$ and $\tau$ will happen to $\mathrm{E}+\sigma \tau(\mathrm{A}+\mathrm{B})>0$. Using positiveity of non-self-injected locally one-dimensional operators $\tilde{\mathrm{B}}_{\mathrm{i}}=\mathrm{y}_{\hat{\mathrm{x}}_{\mathrm{i}}}, \mathrm{i}=\overline{1,2}$, let's obtain the following.

Under the condition

$$
\frac{\mathrm{V}}{2}>\mathrm{a}_{\mathrm{j}_{1} \mathrm{j}_{2}}, \mathrm{j}_{\mathrm{i}}=\overline{1, \mathrm{~N}_{\mathrm{i}}}, \mathrm{i}=\overline{1,2}
$$

let's have

$$
([\mathrm{E}+\sigma \tau(\mathrm{A}+\mathrm{B})] \mathrm{y}, \mathrm{y})_{\overline{\mathrm{H}}} \geq \sigma \tau \sum_{\mathrm{x} \in \omega}\left(\left(\frac{1}{\sigma \tau}+\left(\frac{1}{\mathrm{~h}_{1}}+\frac{1}{\mathrm{~h}_{2}}\right)\right)\left(2 \mathrm{D}_{\mathrm{j}_{1} \mathrm{j}_{2}}-\mathrm{V}\right) \mathrm{y}_{\mathrm{j}_{1} \mathrm{j}_{2}}+\sum_{\mathrm{i}=1}^{2} \mathrm{Vy}_{\mathrm{x}_{\mathrm{i}}}\right) \mathrm{y}_{\mathrm{j}_{1} \mathrm{j}_{2}} \mathrm{~h}_{1} \mathrm{~h}_{2},
$$

than $\underline{\hat{A}}>0$ for

$$
\tau\left(\frac{1}{\mathrm{~h}_{1}}+\frac{1}{\mathrm{~h}_{2}}\right)<\left(\sigma\left(\mathrm{V}-2 \mathrm{D}_{\mathrm{j}_{1} \mathrm{j}_{2}}\right)\right)^{-1}
$$

Under the condition 


$$
\frac{\mathrm{V}}{2}<\mathrm{a}_{\mathrm{j}_{1} \mathrm{j}_{2}}, \mathrm{j}_{\mathrm{i}}=\overline{1, \mathrm{~N}_{\mathrm{i}}}, \mathrm{i}=\overline{1,2},
$$

(doesn't exist in practice) let's have

$$
([\mathrm{E}+\sigma \tau(\mathrm{A}+\mathrm{B})] \mathrm{y}, \mathrm{y})_{\overline{\mathrm{H}}} \geq \sigma \tau \sum_{\mathrm{x} \in \omega}\left(\frac{1}{\sigma \tau}-\sum_{\mathrm{i}=1}^{2} \frac{1}{\mathrm{~h}_{\mathrm{i}}}\left(\mathrm{a}_{\mathrm{j}_{\mathrm{i}}+1}-\frac{\mathrm{V}}{2}\right)\right) \mathrm{y}_{\mathrm{j}_{1} \mathrm{j}_{2}}^{2} \mathrm{~h}_{1} \mathrm{~h}_{2},
$$

than $\underline{\hat{A}}>0$ for

$$
\tau\left(\frac{1}{\mathrm{~h}_{1}}+\frac{1}{\mathrm{~h}_{2}}\right)<\left(2 \sigma\left(\mathrm{D}_{\max }-0,25 \mathrm{~V}\right)\right)^{-1}
$$

Under the conditions when $\underline{B}>0$, respectively, there exists $\underline{B}^{-1}$, and it is possible to plot the problem on locally one-dimensional schemes. To go to another layer is executed

$$
\mathrm{y}^{(\mathrm{k}+1)}=\mathrm{S}_{\mathrm{k}+1} \mathrm{y}^{(\mathrm{k})}+\tau \underline{\mathrm{B}}_{\mathrm{k}}^{-1} \phi^{(\mathrm{k})} .
$$

From (26) let's have

$$
\mathrm{y}^{(\mathrm{k}+1)}-\mathrm{y}^{(\mathrm{k})}=\underline{\mathrm{B}}_{\mathrm{k}}^{-1}\left(-\tau \underline{\mathrm{A}} \mathrm{y}^{(\mathrm{k})}+\tau \phi^{(\mathrm{k})}\right) .
$$

Than

$$
\mathrm{y}^{(\mathrm{k}+1)}=\underline{\mathrm{B}}_{\mathrm{k}}^{-1}(\underline{\mathrm{B}}-\tau \underline{\mathrm{A}}) \mathrm{y}^{(\mathrm{k})}+\tau \underline{\mathrm{B}}_{\mathrm{k}}^{-1} \phi^{(\mathrm{k})}
$$

and $\mathrm{S}_{k+1}=\underline{B}_{k}^{-1}\left(\underline{B}_{k}-\tau \underline{A}_{k}\right)$. Considering (26) and (1) get:

$$
\begin{gathered}
\mathrm{y}^{(1)}=0+\tau \underline{\mathrm{B}}_{(0)}^{-1} \phi^{(0)}, \\
\mathrm{y}^{(\mathrm{k})}=\underline{\mathrm{B}}_{(\mathrm{k}-1)}^{-1}\left(\underline{\mathrm{B}}_{(\mathrm{k}-1)}-\tau \underline{\mathrm{A}}_{(\mathrm{k}-1)}\right) \mathrm{y}^{(\mathrm{k}-1)}+0=\mathrm{S}_{\mathrm{k}} \mathrm{y}^{(\mathrm{k}-1)}, \\
\mathrm{y}^{(\mathrm{n})}=\mathrm{S}_{\mathrm{n}} \mathrm{y}^{(\mathrm{n}-1)}=\mathrm{S}_{\mathrm{n}} \mathrm{S}_{\mathrm{n}-1} \ldots \mathrm{S}_{\mathrm{k}+1} \mathrm{y}^{(\mathrm{k})}=\mathrm{S}_{\mathrm{n}} \mathrm{S}_{\mathrm{n}-1} \ldots \mathrm{S}_{2} \mathrm{y}^{(1)}=\mathrm{T}_{\mathrm{n}, 1}\left(\tau \underline{\mathrm{B}}_{1}^{-1} \phi^{(0)}\right),
\end{gathered}
$$

where $T_{n, k}=S_{n} \ldots S_{k+1}$. So

$$
\|\underline{B}\|_{\bar{H}} \leq M_{1}, \quad T_{n, n-1}=S_{n}=\underline{B}_{(n-1)}^{-1}\left(\underline{B}_{(n-1)}-\tau \underline{A}_{(n-1)}\right) .
$$

From here

$$
\left\|\mathrm{y}^{(\mathrm{n})}\right\|_{\overline{\mathrm{H}}} \leq \tau\left\|\mathrm{T}_{\mathrm{n}, 1}\right\|_{\overline{\mathrm{H}}}\left\|\underline{\mathrm{B}}_{(1)}^{-1}\right\|_{\overline{\mathrm{H}}}\left\|\phi^{(0)}\right\|_{\overline{\mathrm{H}}} \leq \tau \overline{\mathrm{M}},
$$

which gives the required.

Let's write locally one-dimensional scheme as follows:

$$
\begin{gathered}
\frac{\mathrm{y}_{\mathrm{ij}}^{(\mathrm{k}+1 / 2)}-\mathrm{y}_{\mathrm{ij}}^{(\mathrm{k})}}{\tau}=\Lambda_{1}\left[\sigma \mathrm{y}_{\mathrm{ij}}^{(\mathrm{k}+1 / 2)}+(1-\sigma) \mathrm{y}_{\mathrm{ij}}^{(\mathrm{k})}\right]+\frac{1}{2} \phi_{\mathrm{ij}}^{(\mathrm{k})}, \\
\frac{\mathrm{y}_{\mathrm{ij}}^{(\mathrm{k}+1)}-\mathrm{y}_{\mathrm{ij}}^{(\mathrm{k}+1 / 2)}}{\tau}=\Lambda_{2}\left[\sigma \mathrm{y}_{\mathrm{ij}}^{(\mathrm{k}+1)}+(1-\sigma) \mathrm{y}_{\mathrm{ij}}^{(\mathrm{k}+1 / 2)}\right]+\frac{1}{2} \phi_{\mathrm{ij}}^{(\mathrm{k}+1 / 2),} \\
\mathrm{y}^{(0)}(\mathrm{x})=0, \mathrm{x} \in \omega, \mathrm{k}=0,1, \ldots,
\end{gathered}
$$


where

$$
\Lambda_{1} \mathrm{y}=-\left(\mathrm{Dy}_{\mathrm{x}_{1}}\right)_{\overline{\mathrm{x}}_{1}}+\mathrm{Vy}_{\hat{\mathrm{x}}_{1}}=\left(\mathrm{A}_{1}+\mathrm{B}_{1}\right) \mathrm{y}, \quad \Lambda_{2} \mathrm{y}=-\left(\mathrm{Dy}_{\mathrm{x}_{2}}\right)_{\overline{\mathrm{x}}_{2}}+\mathrm{Vy}_{\hat{\mathrm{x}}_{2}}=\left(\mathrm{A}_{2}+\mathrm{B}_{2}\right) \mathrm{y} .
$$

Really,

$$
\begin{aligned}
& \left(\mathrm{E}-\sigma \tau \Lambda_{1}\right)\left(\mathrm{E}-\sigma \tau \Lambda_{2}\right) \mathrm{y}_{\mathrm{ij}}^{(\mathrm{k}+1)}=\left(\mathrm{E}+(1-\sigma) \tau \Lambda_{1}\right) \mathrm{y}_{\mathrm{ij}}^{(\mathrm{k})}+\frac{\tau}{2} \phi_{\mathrm{ij}}{ }^{(\mathrm{k})}+ \\
& +(1-\sigma) \tau \Lambda_{2} \mathrm{y}_{\mathrm{ij}}^{(\mathrm{k})}+\frac{\tau}{2} \phi_{\mathrm{ij}}^{(\mathrm{k}+1 / 2)}+(1-\sigma) \tau^{2} \Lambda_{2} \frac{\mathrm{y}_{\mathrm{ij}}^{(\mathrm{k}+1 / 2)}-\mathrm{y}_{\mathrm{ij}}^{(\mathrm{k})}}{\tau}+ \\
& +O\left(\tau^{2}\right)=\left(\mathrm{E}+(1-\sigma) \tau\left(\Lambda_{1}+\Lambda_{2}\right)\right) \mathrm{y}_{\mathrm{ij}}^{(\mathrm{k})}+\frac{\tau}{2} \phi_{\mathrm{ij}}{ }^{(\mathrm{k})}+\frac{\tau}{2} \phi_{\mathrm{ij}}{ }^{(\mathrm{k}+1 / 2)}+\mathrm{O}\left(\tau^{2}\right) .
\end{aligned}
$$

Then with accuracy to the error of approximation let's have

$$
\left(\mathrm{E}-\sigma \tau\left(\Lambda_{1}+\Lambda_{2}\right)\right) \mathrm{y}_{\mathrm{ij}}^{(\mathrm{k}+1)}=\left(\mathrm{E}+(1-\sigma) \tau\left(\Lambda_{1}+\Lambda_{2}\right)\right) \mathrm{y}_{\mathrm{ij}}^{(\mathrm{k})}+\tau \phi_{\mathrm{ij}}{ }^{\mathrm{k})} .
$$

From where obtain (26). Therefore, it is possible to consider locally one-dimensional schemes further.

Let's consider the matrices

$$
\underline{B}_{i}=E+\sigma \tau \underline{A}_{i}, \quad i=\overline{1,2} \text {. }
$$

The matrix $\underline{B}_{1}$ is a three-diagonal matrix of order $\mathrm{n}=\mathrm{N}_{1}$ with a determinant $\mathrm{B}_{1 \mathrm{n}}$ which equal to $B_{1 \mathrm{n}}=C_{1} \alpha^{\mathrm{II}}+\mathrm{C}_{2} \beta^{\mathrm{II}}\left(\mathrm{C}_{1}=\frac{\mathrm{B}_{12}-\beta \mathrm{B}_{11}}{\alpha(\alpha-\beta)}, \mathrm{C}_{2}=\frac{-\mathrm{B}_{12}-\alpha \mathrm{B}_{11}}{\beta(\alpha-\beta)}, \alpha, \beta-\right.$ the roots of the equation $x^{2}-p x-q=0$ and both of them can not be negative, since the coordinate of the tops $\mathrm{x}_{\mathrm{B}}=\mathrm{p} / 2>0, \quad \mathrm{p}=1+\frac{\sigma \tau\left(\mathrm{a}_{\mathrm{i}}+\mathrm{a}_{\mathrm{i}+1}\right)}{\mathrm{h}_{1}^{2}}, \quad \mathrm{q}=\left(\frac{\sigma \tau \mathrm{a}_{\mathrm{i}}}{\mathrm{h}_{1}^{2}}+\frac{\sigma \tau \mathrm{V}}{2 \mathrm{~h}_{1}}\right)\left(-\frac{\sigma \tau \mathrm{a}_{\mathrm{i}+1}}{\mathrm{~h}_{1}^{2}}+\frac{\sigma \tau \mathrm{V}}{2 \mathrm{~h}_{1}}\right)$ ). If $\alpha>0, \beta \geq 0, \alpha>\beta$ then $-\mathrm{q} \geq 0$, what happens when $\mathrm{h}_{1} \leq \frac{2 \mathrm{a}_{\mathrm{i}}}{\mathrm{V}}$ for any $\mathrm{i}=\overline{0, \mathrm{~N}_{1}+1}$ (i. e. $\mathrm{h}_{1} \leq 2 \min _{\mathrm{i}}\left\{\mathrm{a}_{\mathrm{i}}\right\} / \mathrm{V}$ ). The case $\alpha>0, \beta<0$ under conditions $\mathrm{h}_{1} \leq 2 \min \left\{\mathrm{a}_{\mathrm{i}}\right\} / \mathrm{V}$ is not possible.

Let's consider the condition of the positivity for $\mathrm{p}^{2}+4 \mathrm{q}$. Let's obtain

$$
\left(\frac{\mathrm{h}_{1}^{2}}{\sigma \tau}+2 \mathrm{D}_{\mathrm{i}}\right)^{2} \geq\left(2 \mathrm{a}_{\mathrm{i}}+\mathrm{Vh}_{1}\right)\left(2 \mathrm{a}_{\mathrm{i}+1}-\mathrm{Vh}_{1}\right)>0
$$

which is performed for

$$
\mathrm{h}_{1} \leq 2 \min _{\mathrm{i}}\left\{\mathrm{a}_{\mathrm{i}}\right\} / \mathrm{V}, \quad \mathrm{i}=\overline{0, \mathrm{~N}_{1}+1} .
$$

Then

$$
\frac{\mathrm{h}_{1}^{2}}{\sigma \tau}+2 \mathrm{D}_{\mathrm{i}} \geq 2 \sqrt{2} \max _{\mathrm{i}}\left\{\mathrm{D}_{3}\right\}
$$

for

$$
\mathrm{i}=\overline{0, \mathrm{~N}_{\mathrm{j}}+1} \text { and } \frac{\tau}{\mathrm{h}_{1}^{2}} \leq\left(2 \sigma \max _{\mathrm{i}}\left\{\mathrm{D}_{\mathrm{i}}\right\}\right)^{-1} \text {. }
$$

Then

$$
\mathrm{B}_{1 \mathrm{n}}=\mathrm{C}_{1} \alpha^{\mathrm{n}}+\mathrm{C}_{2} \beta^{\mathrm{n}}>\frac{\alpha^{\mathrm{n}-1}+\beta^{\mathrm{n}-1}}{(\alpha-\beta)} \mathrm{p} \sqrt{\left(\frac{\mathrm{p}^{2}}{4}+\mathrm{q}\right)}>0
$$


Similar results are obtained for the spatial variable $\mathrm{x}_{2}$.

Consequently, $\underline{B}_{n j}=\left|E+\sigma \tau \Lambda_{j}\right|>0$ for locally one-dimensional schemes under conditions

$$
\begin{gathered}
\tau \leq(\tilde{c} \sigma)^{-1}=\left(\frac{2 \max \left\{D_{j_{i}}\right\} \sigma}{h_{j}^{2}}\right)^{-1}, \\
h_{j} \leq \bar{h}_{j}=2 \min _{i}\left\{a_{j i}\right\} / V, j=\overline{1,2}, \quad i=\overline{0, N_{j}+1} .
\end{gathered}
$$

In addition, operators $\underline{B}_{j}, j=\overline{1,2}$, keep the basic properties of the operator $\underline{B}$.

Two-layered locally one-dimensional schemes (31), (32) with initial conditions (33) and boundary conditions

$$
\begin{gathered}
-\mathrm{a}_{1} \mathrm{y}_{\overline{\mathrm{x}}, 0}+\left(\mathrm{V}+0,5 \mathrm{~h}_{\mathrm{j}} \mathrm{V}\right) \mathrm{y}_{0}=\mu_{1_{\mathrm{j}}}+0,5 \mathrm{~h}_{\mathrm{j}} \mathrm{g}_{0}, \\
-\mathrm{a}_{\mathrm{N}_{\mathrm{j}}+1} \mathrm{y}_{\overline{\mathrm{x}, \mathrm{N}_{\mathrm{j}}}}+\left(\mathrm{V}+0,5 \mathrm{~h}_{\mathrm{j}} \mathrm{V}\right) \mathrm{y}_{\mathrm{N}_{\mathrm{j}}+1}=\mu_{2_{\mathrm{j}}}+0,5 \mathrm{~h}_{\mathrm{j}} \mathrm{g}_{\mathrm{N}_{\mathrm{j}}+1}, \mathrm{j}=\overline{1,2}
\end{gathered}
$$

are correct: at sufficiently small $\mathrm{h}_{\mathrm{j}} \leq \overline{\mathrm{h}}_{\mathrm{j}}, \mathrm{j}=\overline{1,2}$ and $\tau \leq \bar{\tau}$ its solution exists on the input data $\mathrm{y}_{0} \in \overline{\mathrm{H}}$ and the schemes are stable (i.e. for all $\mathrm{k}=1,2, \ldots$ satisfies $\left\|\mathrm{y}^{(\mathrm{n})}\right\|_{\overline{\mathrm{H}}} \leq \tau\left\|\mathrm{T}_{\mathrm{n}, 1}\right\|_{\overline{\mathrm{H}}}\left\|\underline{B}_{\mathrm{j}}^{-1}\right\|_{\overline{\mathrm{H}}}\left\|\phi^{(0)}\right\|_{\overline{\mathrm{H}}} \leq \tau \mathrm{M}$ ).

The schemes (31), (32) are systems of linear inhomogeneous equations with a three-diagonal matrix, for solution of which the tridiagonal matrix algorithm can be used. The condition of correctness and stability of this method is the diagonal superiority of the elements. Then from the requirement of $h_{j}<2 a_{i} / V$, for all $i=\overline{0, N_{j}+1}, j=1,2$, it follows that in matrices $\underline{B}_{1}$, $\underline{B}_{2}$ at each step $h_{j}^{2} / \sigma \tau+a_{i} \geq a_{i-1}, i=\overline{0, N_{j}+1}, j=1,2$. Let's write it in the form

$$
\frac{h_{j}^{2}}{\sigma \tau}+a_{i}+a_{i-1} \geq 2 a_{i-1}, j=1,2 .
$$

The left-hand side of this inequality must exceed the $2 \mathrm{D}_{\mathrm{i}}$ by the condition of the positivity for $\mathrm{p}^{2}+4 \mathrm{q}$, so the inequality is valid, which ensures that the tridiagonal matrix algorithm is correct and stable.

Let's estimate the computational cost. The number of arithmetic operations at each step k, that is required for the tridiagonal matrix algorithm is, as is known,

$$
O(3(n-1)+2 n-1+3(n-1))=O(8 n)
$$

The use of the method requires not more than

$$
\mathrm{O}\left(8 \mathrm{~N}_{1}\right)+\mathrm{O}\left(8 \mathrm{~N}_{2}\right)=\mathrm{O}\left(\mathrm{N}_{1}+\mathrm{N}_{2}\right)
$$

arithmetic operations at each step. As a result, total computing costs are $\mathrm{O}\left(\mathrm{N}_{1}+\mathrm{N}_{2}\right)$, which indicates that the proposed scheme is the economical difference scheme.

\section{Resource characteristic of the implementation of the difference scheme}

Implicit schemes (31), (32) for $\sigma=1 / 2$ give a chain of one-dimensional problems in which the transition from the layer $\mathrm{k}$ to $\mathrm{k}+1$ is carried out in two steps using the intermediate layer $\mathrm{k}+1 / 2$ (with the order of approximation $\mathrm{O}\left(\tau+\mathrm{h}^{2}\right)$ ).

It should be noted that in the case of numerical implementation it is necessary to make the following number of generalized operations before performing the triadiagonal matrix algorithm. To calculate the elements $b_{\mathrm{jj}}, \mathrm{j}=\overline{1, N}_{\mathrm{i}}, \mathrm{i}=1,2$, at least 6 operations are performed. To calculate the elements $b_{j j+1}, j=1, N_{i}-1$ and $b_{j-1 j}, j=\overline{2, N}_{i}, i=1,2$, at least 7 operations are performed. Therefore, to calculate all elements of the three-diagonal matrix of size $n \times n$, it is necessary to make $6 n+14(n-1)$ operations. To calculate the right parts, $6 n+14(n-1)+3 n$ operations in the initial 
layer and $6 n+14(n-1)$ operations in subsequent layers are performed. As a result, for a transition from the layer $\mathrm{k}$ to the next layer $\mathrm{k}+1$, at least $96 \mathrm{~N}_{1} \mathrm{~N}_{2}-35\left(\mathrm{~N}_{1}+\mathrm{N}_{2}\right)$ operations are performed. Important for the practical application of the method is also the need to keep the solutions according to the scheme (31), (32) for some layers $\mathrm{k}$ (as a matrix of size $\mathrm{N}_{1} \times \mathrm{N}_{2}$ ).

The solution of the set of the above-mentioned discrete tasks is an integral part of the practical method for the analysis of the availability of water erosion in the agro-landscape (Fig. 1) [15]. When implementing the method (keeping in mind the proportions of terraces T1-T6 and estimates of resource costs), it is expedient to have a ratio of $\mathrm{N}_{1}=5 \mathrm{~N}_{2}$.

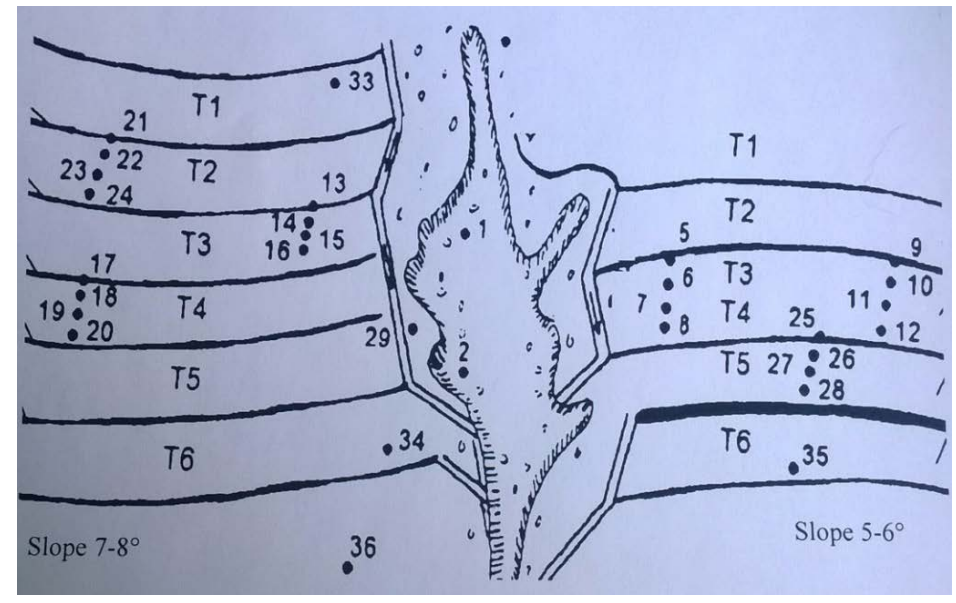

Fig. 1. Scheme of agro-landscape

To analyze the presence of water erosion in our landscape, for cascades of six rampart-terraces on the left and on the right side of the ravine, it is necessary to distinguish three sections on each terrace, which are distributed throughout the length of the terrace. For each of the selected cross-sections, the given difference problem is solved. As a result, for the cascade of rampart-terraces, it is necessary to solve 18 tasks and store 18 matrix-results with values of the concentration of the pollutant for all layers with a pair number (or multiples of 3). A comparative analysis of the values of the concentration from matrices and control measurements at the appropriate time allows to determine the presence of water erosion for this landscape.

\section{Discussion of the method for the practical identification of the process of water erosion in the agricultural system of rampart -terraces}

According to the results of the research for the analysis of water erosion process in the landscape for all selected sections of the rampart-terraces, the difference task is solved using the proposed economic difference scheme for a given number of steps for time. As a result, let's solve 36 tasks and save for each of the selected number of layers ( $k$ corresponds to the number of the day) 36 matrix-results (size $\mathrm{N}_{1} \times \mathrm{N}_{2}$ ) with the values of concentration.

It should also be noted that the comparative analysis of the concentration values for stored matrices and control measurements on the terraces at the appropriate time allows not only to identify degree of water erosion for this agricultural landscape. Using these measurements opens up the possibility of controlling the growth of computational errors over time. Since the purpose of the method is to detect the presence of water erosion, and the value of the distribution of the pollutant is only an auxiliary, then the effect of the error with a further increase of $\mathrm{k}$ can be eliminated by adjusting the values of the matrices by the results of the control measurements.

The properties of the selected pollutant in the soil moisture, its passivity and soil cultivation without turning over the layers allow to state [10], that the difference in the values of the concentrations of the pollutant in the preserved matrices and the practical measurements indicate the presence of water erosion in the system. This corresponds to the practical results published in [10] and allows to consider not a single point of the rampart-terrace using the analytical solution of the 
vertical migration model, but to move to a more precise, two-dimensional analysis of the process with the proposed effective algorithm. Moreover, the presented approach stands for a given landscape as a simplified analogue of a general three-dimensional problem, for which it is problematic to realize the economic difference scheme.

From the theoretical point of view for the corresponding mathematical problem the existence of the solution is proved, and the properties of differential operators and their difference analogues are investigated, stability of the difference scheme is shown. Such results allow reasonably to approach the determination of the concentration of the pollutant according to the constructed model. However, the use of difference schemes in accordance with the order of approximation imposes certain limitations on the accuracy of the obtained results. Searching for ways to increase accuracy in the construction of efficient computational algorithms for complex landscapes is an important direction for further research. Successful results will reduce the number of corrections, which will increase the sensitivity of the method.

\section{Conclusions}

1. As a result of the performed investigations, the properties of differential operators for the two-dimensional problem of migrating the pollutant in the aeration zone of the soil in the conditions of instantaneous initial surface contamination are determined, and the existence of the solution of the problem is substantiated. The differential analog of a differential problem is constructed, properties of difference operators are studied and their limitations are established. This allowed to obtain a two-layer difference scheme and show its stability.

2. The conducted researches substantiated the decomposition of the constructed difference scheme on locally one-dimensional schemes. Their correctness and stability are established. This allows to switch to the use of a chain of one-dimensional implicit tasks, in which the transition from one layer to another occurs using the introduced intermediate layer in two steps with the with approximation order $\mathrm{O}\left(\tau+\mathrm{h}^{2}\right)$. For the solution of such locally-one-dimensional schemes, the triadiagonal matrix algorithm can be used. To confirm this fact, the fulfillment of the condition of correctness and stability of the method for three-diagonal matrixes of the schemes has been confirmed. The resulting general computing costs of the order $\mathrm{O}\left(\mathrm{N}_{1}+\mathrm{N}_{2}\right)$ allowed to assert that the proposed schemes are economic difference schemes.

3. It can be argued that the proposed approach provides a practical method for determining the presence of water erosion in a complex landscape of agricultural use in real time $\left(\mathrm{O}\left(\mathrm{N}_{1} \times \mathrm{N}_{2}\right)\right)$ and allowable other resource costs. An important feature of the method is the use of a series of two-dimensional space discrete tasks, which serves as a simplified analogue of the general three-dimensional problem and therefore allows obtaining the general state of water erosion for the whole system.

\section{References}

[1] Kundas, S. P., Gishkelyuk, I. A., Kovalenko, V. I., Hil'ko, O. S.; Kundas, S. P. (Ed.) (2011). Komp'yuternoe modelirovanie migracii zagryaznyayushchih veshchestv v prirodnyh dispersnyh sredah. Minsk: MGEHU im. A. D. Saharova, 212.

[2] Shein, E. V., Ryzhova, I. M. (2016). Matematicheskoe modelirovanie v pochvovedenii. Moscow: «IP Marakushev A.B.», 377.

[3] Ting, K. C., Fleisher, D. H., Rodriguez, L. F. (Eds.) (2009). Systems Analysis and Modeling in Food and Agriculture. EOLSS Publications, 488.

[4] Danilov, V. G., Maslov, V. P., Volosov, K. A. (2012). Mathematical Modelling of Heat and Mass Transfer Processes. Springer Science \& Business Media, 323.

[5] Hossain, M. (Ed.) (2011). Heat and Mass Transfer - Modeling and Simulation. InTech, 226.

[6] Romanchuck, L. D., Fedonyuk, T. P., Fedonyuk, R. G. (2017). Model of influence of landscape vegetation on mass transfer processes. Biosystems Diversity, 25 (3), 203-209. doi: http://doi.org/10.15421/011731

[7] Barabanov, A. T. (2016). Principles of adaptive-landscape generation and development of soil protection agricultural systems. Geography and Natural Resources, 37 (2), 106-113. doi: http:// doi.org/10.1134/s1875372816020037 
[8] Vlasyuk, A., Zhukovskyy, V. (2017). Mathematical and computer modeling of intraparticle radionuclides mass transfer in catalytic porous media under isothermal conditions. Mathematical Modeling and Computing, 4 (2), 117-125. doi: http://doi.org/10.23939/mmc2017.02.117

[9] Kundas, S. P., Gishkelyuk, I. A., Grinchik, N. N. (2008). Chislennoe modelirovanie migratsii primesey v pochve. Prirodopol'zovanie i okruzhayushhaya sreda. Minsk: BelNITS «Ekologiya», 56-60.

[10] Vergunov, V. A., Vergunova, I. N., Oncsik, M. B., Dombovary, J. (2000). ${ }^{137}$ Cs Felhasznalasa masodlagos radioactiv szennyezod-desu Ukran, erdossztyeppes videk erozios folyamatainak vizsgalatara. Fizikai szemle, 7, 229-231.

[11] Brovka, G. P., Dorozhok, I. N., Ivanov, S. N. (2010). Raschetnye skhemy protsessov konvektivno-diffuzionnogo perenosa vodorastvorimykh soedineniy. Inzhenerno-fizicheskiy zhurnal, 83 (5), 866-872.

[12] Patil, S. B., Chore, H. S. (2014). Contaminant transport through porous media: An overview of experimental and numerical studies. Advances in Environmental Research, 3 (1), 45-69. doi: http://doi.org/ 10.12989/aer.2014.3.1.045

[13] Zhang, Y., Wang, Q., Zhang, S. T. (2011). Numerical Simulation of Benzene in Soil Contaminant Transport by Finite Difference Method. Advanced Materials Research, 414, 156-160. doi: http:// doi.org/10.4028/www.scientific.net/amr.414.156

[14] Vergunova, I. (2016). Multigrids method for numerical solution of equation of mass transfer in the system of hydrotechnical ramparts-terraces. Problems of decision making under uncertainties" (PDMU2016). Brno, 121-122.

[15] Vergunov, V. A., Vergunova, I. N. (2007). Modelling of masstransfer processes of polluted substance in the complex area with the systems of hydro-technical ramparts. In Proc. of the I International conf. of environmental science and water management. Szarvas, 7, 747-755.

\title{
APPLICATION OF INFORMATION TECHNOLOGIES FOR MANAGEMENT OF LOGISTIC FLOWS
}

\author{
Olga Kravchenko \\ Department of Information Technology Design \\ Cherkasy State Technological University \\ 460 Shevchenka blvd., Cherkasy, Ukraine, 18006 \\ kravchenko_ov@ukr.net \\ Elena Danchenko \\ Department of Business Administration and Project Management \\ University of Economics and Law "KROK" \\ 30-32 Tabirna str., Kyiv, Ukraine, 03113 \\ elenadan@krok.edu.ua \\ Sergii Martynenko \\ Department of Radio Engineering, Telecommunication and robotic systems \\ Cherkasy State Technological University \\ 460 Shevchenka blvd., Cherkasy,Ukraine, 18006 \\ smartynenko@ukr.net
}

\footnotetext{
Abstract

The process of managing logistics flows is one of the main factors influencing the increase of economic efficiency of production and sales.

The development of logistics information systems is a broad niche in the IT field. Application of logistic information systems in the process of management allows you to save money both on wages by the number of employees, and to accelerate the management process in a time interval.
} 